\title{
HNSCC and Immunotherapy: The Beginning of a Long Story
}

Thibault Gauduchon*, Thibaut Reverdy, Andy Karabajakian, Laetitia Collet, Max Gau, Eve-Marie Neidhardt and Jérôme Fayette

Department of Medical Oncology, Léon-Bérard Cancer Center, France

*Corresponding author: Gauduchon T, Department of Medical Oncology, Léon-Bérard Cancer Center, France, Tel: +33478782828; E-mail: Thibault.GAUDUCHON@lyon.unicancer.fr

Rec Date: January 17, 2019, Acc Date: February 08, 2019, Pub Date: February 12, 2019

Copyright: @ 2019 Gauduchon T, et al. This is an open-access article distributed under the terms of the Creative Commons Attribution License, which permits unrestricted use, distribution, and reproduction in any medium, provided the original author and source are credited.

\begin{abstract}
The immunotherapy revolution has not spared Head and neck squamous cell carcinoma (HNSCC). Checkpoint inhibitors (anti-PD-1 / PD-L1) were the first to be validated in second line treatment and soon come to the first line in local or metastatic recurrence. Many studies are currently underway to expand the indications of these new therapies, whether as monotherapy or in combination. In addition, immunotherapy is not limited to checkpoint inhibitors and many other immunotherapy molecules are currently under study. The selection of patients who benefit from immunotherapy and the evaluation of the response to these treatments are problems not completely solved. The aim here is to present the state of art on immunotherapy in HNSCC.
\end{abstract}

Keywords: Head and neck squamous cell carcinoma; Checkpoint inhibitors; Immunotherapy; Clinical trial

\section{Introduction}

Head and neck squamous cell carcinoma (HNSCC) is still a public health problem with more than 700000 cases of HNSCC in the world responsible for more than 350000 deaths [1]. About half of the patients will relapse despite heavy treatment combining surgery, radiotherapy and chemotherapy [2]. First- and second-line palliative treatments were only based until recently on chemotherapy and antiEGFR. Such as lung tumor or melanoma, immunotherapy has begun to take its place in the therapeutic arsenal of HNSCC. Indeed, HNSCC are immunosuppressive tumors bypassing the immune system of the host by inducing tolerance of the body. These cancers are therefore particularly sensitive to immunotherapy. We will present here in which situation the immunotherapy was validated in first and second line, then we will see in which situations the immunotherapy is currently under study before approaching the new modalities of immunotherapy under development and finally discuss assessment difficulties with these new therapies.

\section{Validated Treatment in Second Line}

The Checkmate 141 study was the first to lead to an FDA approbation of nivolumab showing an improvement in overall survival in HNSCC progressing after a first platinum-based line. A 32\% reduction in the risk of death $(\mathrm{HR}=0.68$; 95\% CI 0.54-0.86) was observed with an overall survival of 7.7 months versus 5.1 compared with monotherapy with docetaxel, methotrexate or cetuximab $[3,4]$. This difference was greater in PD-L1 patients $>1 \%$ with an overall survival of 8.2 months versus 4.7 , and since curve are separated for PD-L1 negative patients $(<1 \%)$, nivolumab is approved regardless the expression of PD-L1.

Keynote $0-40$ is a second phase III study comparing pembrolizumab in the same situation (but docetaxel was administered every 3 weeks $\left(\mathrm{q} 3 \mathrm{w}\right.$ ) at $75 \mathrm{mg} / \mathrm{m}^{2}$ ). Whereas the first analyze was not significant, the actualization reached statistical significance: the median overall survival was 8.4 months (95\% CI 6.4-9.4) vs. 6.9 (95\% CI 5.9-8.0), $\mathrm{HR}=0.80$ (95\% CI $0.65-0.98, \mathrm{p}=0.0161)$. The reduced HR compared to nivolumab can be explained by i) a cross-over effect in the chemotherapy arm since a large part of patients received subsequently immunotherapy; ii) more patients received docetaxel, which is the most efficient drug with less difference with immunotherapy; iii) $\mathrm{q} 3 \mathrm{w}$ docetaxel is probably more efficient than $\mathrm{q} 1 \mathrm{w}$ of the nivolumab study. Subgroup analyzes showed that in patients $>50 \%$ PD-L1, survival was significantly increased from 7.9 to 11.6 months with nivolumab whereas there is no difference in the $\mathrm{PD}-\mathrm{L} 1<50 \%$ population. These results led to approval in Europe of pembrolizumab in patients whose tumor are $\mathrm{PD}-\mathrm{L} 1>50 \%[5]$.

Another phase III study compared an anti-PD-L1 (durvalumab) alone or with an anti-CTLA4 (Tremelimumab) to a standard chemotherapy. A press release in December announced that the trial was negative for the overall survival.

\section{First Line Treatment}

Keynote 048 is an open-label, randomized, phase 3 study of 882 patients who compared pembrolizumab to the Extreme protocol (cisplatin or carboplatin, 5-fluorouracile (5FU) and cetuximab) in the first line. Pembrolizumab was administered at a fixed dose of $200 \mathrm{mg}$ every 3 weeks alone or in combination with cisplatin and 5FU chemotherapy. Patients were stratified on the expression of PD-L1 $>50 \%$ (by TPS, that is to say the tumor cells). Pre-specified analyses were planned in the whole population and in the populations whose tumor express PD-L1 $\geq 20$ or $\geq 1$ by CPS (a composite score that studies expression on both tumor cells and immune cells in the microenvironment). An interim analysis was presented in October 2018 to ESMO congress [6]. The median overall survival with the pembrolizumab monotherapywas greater than that of Extreme among patients with a CPS score $\geq 20$ with 14.9 vs. 10.7 months ( $\mathrm{HR}=0.61$; $\mathrm{p}=0.0007)$. Among patients with a CPS $\geq 1$ the median survival was 12.3 versus 10.3 months. But it is important to note that if survival is better with pembrolizumab, at the beginning immunotherapy is deleterious for some patients with more deaths in the first six months. There was no difference in PFS between pembrolizumab and Extreme 
in all subgroups with again a cross of the curves and more early progression with pembrolizumab. The tolerability of pembrolizumab is highly better than of Extreme with $17 \%$ of grade 3-5 adverse events versus $69 \%$. Pembrolizumab combined with chemotherapy was non inferior and superior to Extreme for overall survival in total population with a median of 13.0 months versus 10.7 months (and neither significantly better in CPS $\geq 20$ and $\geq 1$ groups in this interim analysis). The response rate with Extreme was $36 \%$ as awaited but was lower with pembrolizumab alone at $23 \%$ in the CPS $\geq 20$ group and $19 \%$ in the CPS $\geq 1$ group). However, the median duration of response is impressive with pembrolizumab at 20.9 months (vs. 4.5 for Extreme). Responses are similar at $36 \%$ for pembrolizumab +chemotherapy.

Pembrolizumab monotherapy is therefore likely to become a firstline standard among patients with a CPS score $\geq 20$ with a selection of patients of the risk of early death who should be treated with chemotherapy. It could be interesting to see if there is a difference among population $\geq 20$ by CPS and $\geq 50$ by TPS. Since the presented results did not separate population CPS $\geq 20$ and CPS $\geq 1$ but $<20$, it is difficult to propose pembrolizumab alone in this population. For the CPS $<20$ population the question arises of chemotherapy alone or with pembrolizumab. Indeed, the data in this population are not available for the benefit of adding Pembrolizumab. This analysis focused only on the total population. It may also be necessary to differentiate the groups CPS $<1, \mathrm{CPS} \geq 1, \mathrm{CPS} \geq 20$. The final results are eagerly awaited.

Two other studies are currently underway on the front line: The KETREL study comparing Extreme with durvalumab alone or with tremelimumab and the CA609-651 study comparing Extreme with a combination of nivolumab and ipilimumab.

\section{Immunotherapy More Precocious}

Since checkpoint inhibitors increase OS after recurrence, could they be of interest in curative intent for localized disease. Trials are in course (immunotherapy compared or added to cisplatin or cetuximab during radiation) and no data of efficacy are available. Today there is no signal of toxicity, but as awaited better tolerance than current treatments. For example, a randomized phase II trial compared radiotherapy potentiated with cetuximab or pembrolizumab among inoperable patients who are ineligible to cisplatin. Tolerance was better in the pembrolizumab arm with $69 \%$ grade $3-4$ toxicity versus $88 \%$ with cetuximab. There was particularly less mucositis and radiodermatitis with pembrolizumab [7].

Immunotherapy could also be added to induction chemotherapy. Phase I is underway that combines durvalumab with TPF (cisplatin docetaxel 5FU).

Still in neoadjuvant, immunotherapy was studied before surgery in untreated patients. A phase 2 trial included 23 patients with probable high-risk HNSCC (probable R1 resection or capsular rupture) to receive pembrolizumab 1 to 3 weeks before surgery: $47 \%$ of response (with an anti-tumor effect on more than $10 \%$ of the surface) was observed, of which $32 \%$ of major response (on more than $70 \%$ of the surface), with 1 complete response and a reduction of $50 \%$ of the awaited high risk tumors after surgery [8]. Similar results have been observed with nivolumab [9]. It is also suggested that immunotherapy could potentially make subsequent chemotherapy more effective. Thus arguing the interest of administering earlier immunotherapy.

\section{News Immunotherapy}

Many studies are currently underway to develop new immunotherapy molecules according to different mechanisms action. A review of the 2016 literature presented the latest insights into the field of immunotherapy for HNSCC [10]. Many immunotherapy associations were already being tested. We will present here the latest news from studies that seem relevant. Table 1 summarizes the molecules presented.

\begin{tabular}{|c|c|c|c|}
\hline Immunotherapy & $\begin{array}{l}\text { Mecanism of } \\
\text { action }\end{array}$ & $\begin{array}{l}\text { Stage of } \\
\text { clinical } \\
\text { develoment }\end{array}$ & Setting \\
\hline M7824 & $\begin{array}{l}\text { Bispecific anti- } \\
\text { PD-L1 and anti- } \\
\text { TGF- } \beta \text { antibody }\end{array}$ & Phase I & $\begin{array}{l}\text { Recurrent/metastatic } \\
\text { HNSCC in second } \\
\text { therapeutic line }\end{array}$ \\
\hline $\begin{array}{l}\text { Monalizumab with } \\
\text { cetuximab }\end{array}$ & $\begin{array}{l}\text { New checkpoint } \\
\text { inhibitor } \\
\text { targeting } \\
\text { NKG2A+anti } \\
\text { EGFR }\end{array}$ & Phase II & $\begin{array}{l}\text { Recurrent/metastatic } \\
\text { HNSCC }\end{array}$ \\
\hline $\begin{array}{l}\text { Danvatirsen with } \\
\text { durvalumab }\end{array}$ & $\begin{array}{l}\text { Inhibitory } \\
\text { antisense } \\
\text { oligonucleotide } \\
\text { of STAT3+anti- } \\
\text { PD-L1 }\end{array}$ & Phase Ib/ll & $\begin{array}{l}\text { Metastatic HNSCC } \\
\text { naive of anti-PD-L1 }\end{array}$ \\
\hline $\begin{array}{l}\text { SD101 with } \\
\text { pembrolizumab }\end{array}$ & $\begin{array}{l}\text { TLR9 agonist } \\
\text { +anti-PD-1 }\end{array}$ & Phase I/II & $\begin{array}{l}\text { Recurrent/metastatic } \\
\text { HNSCC, anti-PD1 } \\
\text { naive }\end{array}$ \\
\hline Epacadostat & $\begin{array}{l}\text { Oral } \\
\text { inhibitor }\end{array}$ & $\begin{array}{l}\text { Phase III } \\
\text { failures }\end{array}$ & $\begin{array}{l}\text { Recurrent/metastatic } \\
\text { HNSCC }\end{array}$ \\
\hline Lenvatinib & $\begin{array}{l}\text { Broad-spectrum } \\
\text { TKI }\end{array}$ & Phase Ib/ll & $\begin{array}{l}\text { Metastatic HNSCC, } \\
\text { after at least one line of } \\
\text { chemotherapy }\end{array}$ \\
\hline $\begin{array}{l}\text { MEDI6974 with } \\
\text { durvalumab }\end{array}$ & $\begin{array}{l}\text { Ox40 agonist } \\
\text { antibody+anti- } \\
\text { PD-L1 }\end{array}$ & Phase lb & $\begin{array}{l}\text { Before surgery with } \\
\text { stage III-IVA HNSCC }\end{array}$ \\
\hline
\end{tabular}

Table 1: Recent immunotherapy approaches in HNSCC.

A phase 1 study showed promising results with a bispecific anti-PDL1 and anti-TGF- $\beta$ antibody (M7824). The goal is to inhibit TGF- $\beta$, which increases tumor immunodepression. In addition, inhibition of the TGF- $\beta$ pathway could increase the activity of anti-PD-L1. Among 32 patients, 7 had partial response, 6 had tumor stability, and grade III was found among 10 patients (31.3\%). Further development will be interesting to follow [11].

Monalizumab is a new checkpoint inhibitor targeting NKG2A. This is expressed on CD8+lymphocytes and on NK lymphocytes. The ligand of NKG2A, HLA-E, is overexpressed in HNSCC. Blocking this interaction stimulate CD8+ (and NK lymphocytes and increase ADCC (Antibody-dependent cell-mediated cytotoxicity). Since Cetuximab acts mainly via an ADCC mechanism, it could be potentiated by Monalizumab. A phase II study included 40 patients to receive weekly cetuximab combined to monalizumab every two weeks after failur of platin. The response rate, which was the primary endpoint, was $27.8 \%$, or 11 out of 40 patients (compared to $13 \%$ with cetuximab alone according to historical data). The tolerance was particularly good, making this association a promising issue [12]. 
Another phase Ib/II study investigated the combination of danvatirsen with durvalumab among patients with metastatic HNSCC naive of anti-PD-L1. Danvatirsen is an inhibitory antisense oligonucleotide of STAT3 (that stimulates the immunosuppressive function of the microenvironment). Among 44 treated patients, $23 \%$ of responses (higher than those found with durvalumab monotherapy) were found with 3 complete responses. An increase of transaminases and transient thrombocytopenia were the major toxicities and it seems there is no additive toxicity of the two treatments [13].

SD101 is a TLR9 agonist stimulating the activity of dendritic cells. This later became antigen-presenting cells to activate an anti-tumor response via $\mathrm{T}$ lymphocytes. A phase $\mathrm{I} / \mathrm{II}$ study investigated the combination with pembrolizumab. Patients received pembrolizumab every 3 weeks and SD-101 injected into a single tumor site each week for 4 injections and then every 3 weeks up to 7 injections. Among the 26 patients included, the median injections of SD-101 administered were 5. Patients experienced injection site reactions and influenza-like illness with $28 \%$ of toxicities $\geq$ grade 3 . The immune-induced effects of pembrolizumab do not appear to have increased. The response rate in mITT (that is to say, among the 23 patients who had reached at least the first tumor evaluation) was $30.4 \%$ with 7 partial responses, 3 stabilities, 10 progressions in imaging and 3 clinical progressions before the scan. Tumor size reductions $\geq 30 \%$ were observed in injected lesions (8/18 or $44.4 \%)$ as well as in non-injected lesions $(8 / 14$ or $57.1 \%)$. At the time of the analysis, 5 of the 7 responses were ongoing ( 1 to 27 weeks). Therefore, stimulation of dendritic cells seems promising with activity in the injected site, but also at a distance, at the cost however of a significant toxicity [14].

Epacadostat is an oral IDO1 inhibitor (indoleamine 2,3dioxygenase) showing good phase I and II in combination with pembrolizumab [15]. IDO1 is an enzyme expressed in the tumor microenvironment that has an immunosuppressive effect by decreasing tumor infiltration by cytotoxic T lymphocytes (TILs). But in the face of phase III failures in melanoma, development has unfortunately been halted.

Associations with TKIs have also been studied in a phase Ib/II study. Lenvatinib, a broad-spectrum TKI, has been associated with pembrolizumab in 22 progressive metastatic patients after at least one line of chemotherapy. The 24 -week response rate was $36 \%$ with a median response time of 8.2 months, a PFS of 8.2 months, and one year progression-free survival rate of $37 \%$. But the toxicity is important with $91 \%$ of side effect, of which $14 \%$ of grade 4 and $18 \%$ of patients who had to stop the treatment. The main symptoms were fatigue, loss of appetite, hypertension and digestive disorders. Associations with other less toxic tyrosine kinase inhibitors may be considered [16].

Another trial associated durvalumab with an activating agonist of the immune response, MEDI6974 (Ox40 agonist antibody). The treatments were administered for a short time before surgery. An increase and activation of cytotoxic lymphocytes within the tumor has been demonstrated. This association could potentially be interesting [17].

\section{Difficulty of Assessing Immunotherapy}

Evaluation of immunotherapies may be more difficult than conventional anti-tumor treatments. Indeed, patients treated with ICP observed phenomena of pseudo-progression (tumor response after an initial progression) and, on the contrary, hyperprogression phenomena with a rapid and significant increase in tumor volume.
These phenomena of pseudo progression are rare, but may explain the benefit of immunotherapy treatment beyond progression. For example, in the Checkmate study 141, it was observed that some patients continued treatment despite the RECIST progression and then presented with stability or response (3\% of patients had responded by RECIST after progression). Most of these patients were in good general condition despite the initial progression. It therefore seems that maintaining a good general condition and a certain clinical benefit of treatment during radiological progression are good criteria for selecting these patients. On the contrary, the expression of PDL1 does not seem to differentiate the patients who will be treated or not beyond progression.

This pseudo progression can in part explain the possible increase of efficacy of subsequent chemotherapy after failure of anti-PD-1. Indeed a French retrospective study in 82 patients receiving chemotherapy (45\% monotherapy and 55\% combination therapy) after failure of immunotherapy showed a $30 \%$ responses and a $57 \%$ disease control, higher than those usually observed in the 3rd line. Chemotherapy could also activate the immune system by releasing tumor antigens, with check point inhibitors known to remain in the body for a long time.

Hyperprogressions are associated with poorer survival. A French retrospective study suggested that hyperprogression can be observed in $29 \%$ of patients treated by immunotherapy [18]. This controversial phenomenon could be induced by an excessive immune reaction, a paradoxical action of anti-PD-1/PD-L1 promoting tumor development, or ultimately only the natural history of advanced phase cancer.

Finally, unlike conventional chemotherapy, the occurrence of autoimmune adverse events seems to correlate with the effectiveness of the checkpoint inhibitors. In an analysis of 114 patients treated with anti-PD-1: $43 \%$ exhibited immunological toxicity. These patients had higher response rates (30.6\% versus $12.3 \%)$, better progression-free survival (6.9 months versus 2.1) and improved overall survival (12.5 months vs. 6.8). This analysis may of course be biased since patients who benefit from immunotherapy pursue it longer and are therefore more likely to develop immunological toxicity [19].

\section{Predictive Factor of Efficacy of Immunotherapy}

One of the major challenges of immunotherapy is to select patients responding to immunotherapies with predictive biomarkers of effectiveness. Currently, the only one used is the expression of PDL1 but it is very imperfect, especially with excellent results in PDL1 negative patients.

The expression of PD-L2 and the signature IFNg have been explored, but do not seem particularly convincing.

HNSCC have been classified into 6 groups: immunoreactive, inflammatory, HPV-like, classical, hypoxemic and mesenchymal [20]. The inflammatory and immunoreactive profiles are probably more sensitive to immunotherapies because they are strongly infiltrated by cells of the immune system. The important tumoral infiltration by the CD8 lymphocytes could characterize this inflammatory type.

In a cohort of 34 patients with significan CD8+lymphocyte infiltration and a high PD-1/TIM3 expression ratio (regardless of HPV status and smoking status) showed an increase in overall survival (84 vs. 13 months) with anti-PD-1 treatment, compared with noninflammatory tumors [21]. 
The estimation of the mutational load (TMB) in the primary tumor, but also from the circulating DNA could also become a predictive biomarker [22].

Much simpler, a study presented at ASCO 2018 has recently been shown to have a high lymphocyte neutrophil count associated with tumor inflammation and poor prognosis [23]. 114 patients were treated with anti-PD1 classified in 4 quartiles according to this ratio. Patients with the lowest ratio had an overall survival well above 12.5 months compared to those with the highest ratio (4.1 months, $\mathrm{p}$ $<0.0001)$. In this cohort, PD-L1 expression did not discriminate patients. Thus, a very simple and clinical marker proves to be a powerful prognostic factor for the efficacy of immunotherapies.

\section{Conclusion}

The immunotherapy revolution has not spared head and neck oncology. Indeed, anti PD-1 are now an integral part of the therapeutic arsenal and new indications are emerging either as monotherapy or in combination. Furthermore, immunotherapy is not limited to checkpoint inhibitors and many molecules are under development. However, many questions remain: What is the optimal duration of these immunotherapy treatments? Similarly, how to determine the best treatment combinations? And for which patient? The therapeutic management of HNSCC will certainly be greatly modified in the years to come.

\section{References}

1. Bray F, Ferlay J, Soerjomataram I, Siegel RL, Torre LA, et al. (2018) Global cancer statistics 2018: GLOBOCAN estimates of incidence and mortality worldwide for 36 cancers in 185 countries. CA Cancer J Clin 68: 394-424.

2. Karabajakian A, Toussaint P, Neidhardt EM, Paulus V, Saintigny P, et al. (2017) Chemotherapy for recurrent/metastatic head and neck cancers. Anti-cancer Drugs 28: 357-361.

3. Ferris RL, Blumenschein Jr G, Fayette J, Guigay J, Colevas AD, et al. (2016) Nivolumab for recurrent squamous-cell carcinoma of the head and neck. N Engl J Med 375: 1856-1867.

4. Ferris RL, Blumenschein G, Fayette J, Guigay J, Colevas AD, et al. (2018) Nivolumab vs investigator's choice in recurrent or metastatic squamous cell carcinoma of the head and neck: 2-year long-term survival update of CheckMate 141 with analyses by tumor PD-L1 expression. Oral Oncol 81: 45-51.

5. Cohen EE, Soulières D, Le Tourneau C, Dinis J, Licitra L, et al. (2019) Pembrolizumab versus methotrexate, docetaxel, or cetuximab for recurrent or metastatic head-and-neck squamous cell carcinoma (KEYNOTE-040): a randomised, open-label, phase 3 study. Lancet 393: 156-167.

6. Burtness B, Harrington KJ, Greil R, Soulières D, Tahara M, et al. (2018) KEYNOTE-048: Phase 3 study of first-line pembrolizumab (P) for recurrent/metastatic head and neck squamous cell carcinoma (R/M HNSCC). Oncologypro.

7. Sun XS, Sire C, Tao Y, Martin L, Alfonsi M, et al. (2018) A phase II randomized trial of pembrolizumab versus cetuximab, concomitant with radiotherapy (RT) in locally advanced (LA) squamous cell carcinoma of the head and neck (SCCHN): First results of the GORTEC 2015-01 "PembroRad" trial. J Clin Oncol 36: 6018-6018.

8. Uppaluri R, Zolkind P, Lin T, Nussenbaum B, Jackson RS, et al. (2017) Neoadjuvant pembrolizumab in surgically resectable, locally advanced HPV negative head and neck squamous cell carcinoma (HNSCC). J Clin Oncol 35: 6012.
9. Ferris RL, Gonçalves A, Baxi SS, Martens UM, Gauthier H, et al. (2017) LBA46An open-label, multicohort, phase $1 / 2$ study in patients with virusassociated cancers (CheckMate 358): Safety and efficacy of neoadjuvant nivolumab in squamous cell carcinoma of the head and neck (SCCHN). Ann Oncol 28: 440-441.

10. Economopoulou P, Kotsantis I, Psyrri A (2016) The promise of immunotherapy in head and neck squamous cell carcinoma: combinatorial immunotherapy approaches. ESMO Open 1: e000122.

11. Cho BC, Daste A, Ravaud A, Salas S, Isambert N, et al. (2018) M7824 (MSB0011359C), a bifunctional fusion protein targeting PD-L1 and TGF_, in patients (pts) with advanced SCCHN: results from a phase 1 cohort. Oncologypro.

12. Cohen R, Fayette J, Posner M, Lefebvre G, Bauman J, et al. (2018) Abstract CT158: Phase II study of monalizumab, a first-in-class NKG2A monoclonal antibody, in combination with cetuximab in previously treated recurrent or metastatic squamous cell carcinoma of the head and neck (R/M SCCHN): Preliminary assessment of safety and efficacy. Cancer Res 78: CT158.

13. Cohen EEW, Harrington KJ, Hong DS, Mesia R, Brana I, et al. (2018) A phase Ib/II study (SCORES) of durvalumab (D) plus danvatirsen (DAN; AZD9150) or AZD5069 (CX2i) in advanced solid malignancies and recurrent/metastatic head and neck squamous cell carcinoma (RMHNSCC): Updated results. Ann Oncol 29: 287.

14. Cohen EE, Algazi A, Laux D, Wong DJ, Amin A, et al. (2018) 1050PD Phase $\mathrm{Ib} / \mathrm{II}$, open label, multicenter study of intratumoral SD-101 in combination with pembrolizumab in anti-PD-1 treatment naïve patients with recurrent or metastatic head and neck squamous cell carcinoma (HNSCC). Ann Oncol 29: 287.

15. Mitchell TC, Hamid O, Smith DC, Bauer TM, Wasser JS, et al. (2018) Epacadostat plus pembrolizumab in patients with advanced solid tumors: phase I results from a multicenter, open-label phase I/II trial (ECHO-202/ KEYNOTE-037). J Clin Oncol 36: 3223.

16. Taylor MH, Rasco DW, Brose MS, Vogelzang NJ, Richey SL, et al. (2018) A phase $1 b / 2$ trial of lenvatinib plus pembrolizumab in patients with squamous cell carcinoma of the head and neck. J Clin Oncol 36: 6016.

17. Bell RB, Duhen R, Leidner RS, Curti BD, Ballesteros-Merino C, et al. (2018) Neoadjuvant anti-OX40 (MEDI6469) prior to surgery in head and neck squamous cell carcinoma. J Clin Oncol 36: 6011.

18. Saâda-Bouzid E, Defaucheux C, Karabajakian A, Coloma VP, Servois V, et al. (2017) Hyperprogression during anti-PD-1/PD-L1 therapy in patients with recurrent and/or metastatic head and neck squamous cell carcinoma. Ann Oncol 28: 1605-1611.

19. Foster CC, Kochanny S, Khattri A, Acharya R, Dekker A, et al. (2018) Association of immune-related adverse events (irAEs) with improved response, progression-free survival, and overall survival for patients with metastatic head and neck cancer receiving anti-PD-1 therapy. J Clin Oncol 36: 6014 .

20. De Cecco L, Nicolau M, Giannoccaro M, Daidone MG, Bossi P, et al. (2015) Head and neck cancer subtypes with biological and clinical relevance: Meta-analysis of gene-expression data. Oncotarget 6: 9627.

21. Hanna GJ, Liu H, Jones RE, Bacay AF, Lizotte PH, et al. (2017) Defining an inflamed tumor immunophenotype in recurrent, metastatic squamous cell carcinoma of the head and neck. Oral Oncol 67: 61-69.

22. Khagi Y, Goodman AM, Daniels GA, Patel SP, Sacco AG, et al. (2017) Hypermutated circulating tumor DNA: correlation with response to checkpoint inhibitor-based immunotherapy. Clin Cancer Res 23: 5729-5736.

23. Foster CC, Kochanny S, Khattri A, Acharya R, Dekker A, et al. (2018) Association of a baseline neutrophil-to-lymphocyte ratio (NLR) with progression-free and overall survival in head and neck cancer patients receiving anti-PD-1 therapy. J Clin Oncol 36: 6038. 